\title{
Determinação do coeficiente convectivo de transferência de calor de figos submetidos ao resfriamento rápido
}

\author{
Mariangela Amendola ${ }^{1}$, Saul Dussán-Sarria ${ }^{2} \&$ Anderson A. Rabello ${ }^{3}$
}

\begin{abstract}
RESUMO
Este trabalho busca, como função principal, determinar o valor do coeficiente convectivo de transferência de calor através da metodologia de modelagem matemática e simulação numérica associada ao processo experimental previamente realizado, de resfriamento rápido com ar forçado de figos "Roxo de Valinhos". O objetivo foi comparar o resultado com o definido pelo ajuste entre os dados experimentais e o uso de equação empírica da literatura, já realizado. Para a predição da temperatura próximo ao centro das frutas, considerou-se o modelo da lei de Fourier em coordenadas esféricas e se implementaram algoritmos segundo os métodos de diferenças finitas e dos elementos finitos para os modelos unidimensional e tridimensional, respectivamente. O estabelecimento do referido valor se dá a partir da comparação desses dados simulados com os dados experimentais. Os valores resultantes dos dois modelos foram coincidentes e resultaram menores valores residuais que o obtido pela equação empírica; além disso, a representatividade da curva simulada mostra que a atual metodologia é mais precisa que a anteriormente realizada e, portanto, adequada para este e trabalhos futuros.
\end{abstract}

Palavras-chave: simulação numérica, frutas esféricas, modelo matemático, métodos numéricos

\section{Determination of the convective heat transfer coefficient of fig fruits submitted to forced air precooling}

\begin{abstract}
The main purpose of this study is to determine the convective heat transfer coefficient value by using the methodology of mathematical modeling and numerical simulation associated with the experimental process previously performed, of the forced air precooling of the figs type 'Roxo de Valinhos'. The objective was to compare the result with the one determined by fitting an empirical equation from the literature to the experimental data. For the prediction of the temperature, near to the center of fruits, the Fourier law model was considered. Algorithms employing the finite differences and finite elements methods were implemented for the one-dimensional and three-dimensional models. The establishment of the referred value was performed through the comparison of these simulated data with the experimental data. The resulting values of the two models were coincident and generated a smaller residual value than the one obtained by the empirical equation. Furthermore, the representation of the simulated curve shows that the methodology of the mathematical modeling and numerical simulation is more accurate than the previous one and suitable for this and future work.
\end{abstract}

Key words: numerical simulation, spherical fruits, mathematical model, numerical methods

\footnotetext{
FEAGRI/UNICAMP, Cidade Universitária Zeferino Vaz, s/n, CP 6011, CEP 13083-970, Campinas, SP. Fone: (19) 3521-1065, Fax: (19) $3521-1005$. E-mail: amendola@agr.unicamp.br

2 Facultad de Ingeniería y Administración. Universidad Nacional de Colombia. Sede Palmira, Valle del Cauca, Colombia. E-mail: sauldussan@yahoo.com.br

${ }^{3}$ Departamento de Engenharia Elétrica, Centro Federal de Educação Tecnológica de Minas Gerais, Av. Amazonas n.7675, CEP 30510-000, Belo Horizonte, MG. Fone: (31) 8797-9015. E-mail: arthur@dppg.cefetmg.br
} 


\section{INTRODUÇÃO}

O Brasil é o terceiro maior produtor de frutas do mundo com aproximadamente 44 milhões de toneladas no ano 2004 (FAOSTAT, 2008). O Estado de São Paulo o responsável por cerca de $80 \%$ da produção brasileira de figo e a região de Valinhos a principal produtora (IBGE, 2008).

A perecibilidade das frutas pode ser reduzida se assegurados os procedimentos, tanto do seu resfriamento, imediatamente após a colheita, como do subseqüente armazenamento refrigerado e pode ser maior ou menor, de acordo com o manuseio durante os procedimentos (Turk, 1989; Dincer, 1995).

O resfriamento rápido com ar forçado consiste em fazer passar o ar frio através da pilha de embalagens contendo as frutas. Durante o resfriamento o coeficiente convectivo de transferência de calor se estabelece em função das dimensões das embalagens, da sua área de abertura, da sua forma de distribuição, das características das frutas (temperatura, teor de água, calor específico e forma geométrica), além das características do ar de resfriamento (temperatura e velocidade) (Thompson et al., 1998).

Como consta em Dussán-Sarria (2003), o figo "Roxo de Valinhos”, altamente perecível, é um dos cultivares que apresentam pouca informação bibliográfica no que diz respeito aos parâmetros de transferência de calor; assim, o autor apresenta, em seu trabalho de tese de doutorado, uma metodologia matemática para o estabelecimento do valor do coeficiente convectivo de transferência de calor desta fruta. Apesar dos bons resultados, a confirmação e/ou busca de precisão indica a urgência de comparação entre estes com os obtidos por outra metodologia.

Novy \& Kierckbush (1986) desenvolveram um modelo simples para o cálculo da taxa de resfriamento. Outros modelos foram desenvolvidos por Mascheroni \& Calvelo (1982), nos quais os autores utilizam equações auxiliares em função de características básicas, como entalpia, condutividade térmica, calor específico, difusividade térmica e geometria, dentre outras propriedades e características dos produtos.

Utilizou-se, neste trabalho, a mesma metodologia de modelagem matemática e simulação numérica que vem sendo utilizada em pesquisas da FEAGRI/UNICAMP para determinação de parâmetros físicos de produtos agrícolas. Isto porque esta metodologia se mostrou adequada, conforme revelam os trabalhos de Pirozzi (2002), Amendola (2003a), Amendola (2003b, 2004), Amendola \& Teruel (2005), Pirozzi \& Amendola (2005), e Amendola (2006a, b e c).

Tal adequação justifica o presente estudo que, além de complementar o que constou em Amendola \& Dussán-Sarria (2005), mostra com detalhes o que se constatou em Rabello et al. (2006), ambos os trabalhos realizados com o objetivo de buscar precisão na determinação do coeficiente convectivo de transferência de calor dos frutos.

\section{MATERIAL E MÉTODOS}

Os parâmetros físicos e os dados experimentais de tem- peratura próxima ao centro de frutas submetidas a um tempo total de resfriamento por ar forçado foram considerados de acordo com o que consta no experimento descrito com detalhes em Dussán-Sarria (2003). As frutas são da figueira cultivar Roxo de Valinhos, adquiridas de produtores da região de Valinhos, SP (safra 2002), colhidas no estádio rami (3/4 de maturidade) as quais, uma vez colhidas, foram selecionadas de forma a caracterizar uma amostra homogênea a ser submetida ao processo de resfriamento rápido por ar forçado e, portanto, adequada para receber o tratamento matemático aqui proposto.

No experimento, o valor do raio das frutas (R) foi $0,0285 \mathrm{~m}$, a temperatura inicial das frutas $\left(\mathrm{T}_{0}\right)$ foi $19^{\circ} \mathrm{C}$, a temperatura do ar de resfriamento $\left(\mathrm{T}_{\mathrm{a}}\right) 0^{\circ} \mathrm{C}$ e a umidade relativa do ar em torno de $75 \%$. O teor de água do figo no início do resfriamento foi $84,3 \%$ e, no final do processo, 83,3\%. As frutas foram embaladas em caixa de madeira e utilizadas 48 embalagens, 24 em cada lado, para formar o túnel tipo californiano. A caixa de madeira apresentava uma percentagem de área de abertura de 9,2\%. O fluxo de ar correspondeu a 2,8 $\mathrm{L} \mathrm{s}^{-1} \mathrm{~kg}^{-1}$, com velocidade média do ar entre as frutas de $1,5 \mathrm{~m} \mathrm{~s}^{-1}$. O tempo de resfriamento foi de $100 \mathrm{~min}$.

O coeficiente de difusividade térmica utilizado $(\alpha)$ foi determinado experimentalmente através do método Fonte Linear de calor, cujo valor foi de $0,156 \times 10^{-6} \mathrm{~m}^{2} \mathrm{~s}^{-1}$, o valor da condutividade térmica $\left(\mathrm{k}_{\mathrm{p}}\right)$, também definido experimentalmente, foi de $0,52 \mathrm{~W} \mathrm{~m}^{-1}{ }^{\circ} \mathrm{C}^{-1}$ e o valor do calor específico experimental $\left(\mathrm{C}_{\mathrm{p}}\right)$ foi de $4,07 \mathrm{~kJ} \mathrm{~kg}^{-1}{ }^{\circ} \mathrm{C}^{-1}$ (Dussán-Sarria \& Honório, 2004). Nota-se que o valor da densidade da fruta já está embutido no valor de a. O valor da esfericidade das frutas resultou em $96 \%$.

De acordo com o trabalho prévio de Dussán-Sarria (2003), para determinação do valor do coeficiente convectivo de transferência de calor $\left(\mathrm{h}_{\mathrm{c}}\right)$ tomou-se o conjunto de dados de temperatura adimensional:

$$
\theta=\left(\mathrm{T}-\mathrm{T}_{\mathrm{m}}\right) /\left(\mathrm{T}_{\mathrm{i}}-\mathrm{T}_{\mathrm{m}}\right)
$$

em que:

$\mathrm{T}_{\mathrm{i}}$ - corresponde à temperatura inicial da fruta, ${ }^{\circ} \mathrm{C}$

$\mathrm{T}_{\mathrm{m}}$ - corresponde à temperatura do meio tempo de resfriamento, ${ }^{\circ} \mathrm{C}$

$\mathrm{T}$ - refere-se à temperatura próxima ao centro das frutas, em qualquer instante, ${ }^{\circ} \mathrm{C}$

em seguida, não dispondo de outros recursos matemáticos ou computacionais, empregou-se o programa Excel para o ajuste dos referidos dados ao modelo exponencial:

$$
\theta=\mathrm{Je}^{-\mathrm{at}}
$$

o qual gerou os seguintes valores dos parâmetros de ajuste: $\mathrm{a}=-0,0289$ e $\mathrm{J}=1,1244$, com $\mathrm{R}^{2}=0,99$. O valor do coeficiente convectivo de transferência de calor foi então calculado como uma primeira abordagem, utilizando-se a expressão empírica definida por Dincer (1995):

$$
h_{c}=(3,2 k(2 R) a) /\left(10,3 \alpha-a(2 R)^{2}\right)
$$


resultando no valor $\mathrm{h}_{\mathrm{c}}=18,8 \mathrm{~W} \mathrm{~m}^{-2}{ }^{\circ} \mathrm{C}^{-1}$.

Observa-se, apesar de esta equação (3) ter sido determinada para outro cultivar submetido a condições experimentais diferentes das consideradas neste trabalho, que ela foi a única expressão matemática encontrada na literatura para definir o parâmetro em questão, fazendo-se uso de outros parâmetros de resfriamento, como condutividade e difusividade térmica, justificando a busca de outra abordagem metodológica como a que foi feita no presente trabalho, razão por que para a determinação do valor de $\mathrm{h}_{\mathrm{c}}$ da fruta foram utilizados, neste trabalho, o modelo matemático e os métodos numéricos, como descritos a seguir.

O modelo matemático que descreve o processo experimental se baseia na Lei de Fourier, em que a temperatura $\mathrm{T}=\mathrm{T}(\mathrm{x}, \mathrm{y}, \mathrm{z})$ da fruta, considerando-se as propriedades térmicas constantes, pode ser modelada como:

$$
\frac{\partial \mathrm{T}}{\partial \mathrm{t}}=\alpha \nabla^{2} \mathrm{~T} ; \quad \mathrm{t} \geq 0 ; \quad \mathrm{em} \mathrm{V}
$$

em que:

$$
\begin{aligned}
& \mathrm{t} \text { - tempo, } \mathrm{s} \\
& \alpha \text { - difusividade térmica da fruta, } \alpha=\mathrm{k}_{\mathrm{p}} \rho^{-1} \mathrm{C}_{\mathrm{p}}{ }^{-1} \text {, } \\
& \\
& \mathrm{m}^{2} \mathrm{~s}^{-1} \\
& \rho \text { - densidade da fruta, } \mathrm{kg} \mathrm{m}^{-3} \\
& \mathrm{k}_{\mathrm{p}} \text { - condutividade térmica da fruta, } \mathrm{W} \mathrm{m}{ }^{-1}{ }^{\circ} \mathrm{C}^{-1} \\
& \mathrm{C}_{\mathrm{p}} \text { - calor específico da fruta, } \mathrm{kJ} \mathrm{kg}^{-1}{ }^{\circ} \mathrm{C}^{-1} \\
& \nabla^{2} \text { - operador laplaciano }
\end{aligned}
$$

A condição inicial é dada por:

$$
\mathrm{T}(\mathrm{x}, \mathrm{y}, \mathrm{z}, 0)=\mathrm{T}_{0}
$$

Por um lado, associada à condição de simetria do problema, tem-se a condição de contorno:

$$
\frac{\partial \mathrm{T}}{\partial \mathrm{t}}(0, \mathrm{t})=0 ; \quad \mathrm{t}>0
$$

É fato que o fluxo de calor através da fruta está relacionado aos coeficientes de transferência de calor e massa; no entanto, ao se desconsiderar a transferência de massa por ser muito pequena, a exemplo do que consideram outros autores (Amendola \& Teruel, 2005; Pirozzi \& Amendola, 2005), estabelece-se a seguinte aproximação para a outra condição de contorno:

$$
\kappa_{\mathrm{p}} \frac{\partial \mathrm{T}}{\partial \mathrm{t}}(\mathrm{x}, \mathrm{y}, \mathrm{z}, \mathrm{t})=\mathrm{h}_{\mathrm{c}}\left[\mathrm{T}_{\mathrm{s}}(\mathrm{t})-\mathrm{T}_{\mathrm{a}}\right] ; \mathrm{t} \geq 0
$$

sendo:

$\mathrm{h}_{\mathrm{c}}$ - coeficiente de transferência de calor por convecção, $\mathrm{W} \mathrm{m} \mathrm{m}^{-2}{ }^{\circ} \mathrm{C}^{-1}$

$\mathrm{T}_{\mathrm{s}}$ - temperatura na superfície da fruta, ${ }^{\circ} \mathrm{C}$

$\mathrm{T}_{\mathrm{a} \text { - }}$ temperatura na câmara de resfriamento, ${ }^{\circ} \mathrm{C}$

O modelo unidimensional é o já utilizado nas referidas pesquisas precedentes da FEAGRI/UNICAMP que, após ser adaptado de Trelea et al. (1998), escrito em coordenadas esféricas, resulta na equação para determinação da tempe- ratura $T=T(r, t)$ de frutas esféricas:

$$
\frac{\partial \mathrm{T}}{\partial \mathrm{t}}(\mathrm{r}, \mathrm{t})=\alpha\left(\frac{2}{\mathrm{r}} \frac{\partial \mathrm{T}}{\partial \mathrm{r}}(\mathrm{r}, \mathrm{t})+\frac{\partial^{2} \mathrm{~T}}{\partial \mathrm{r}^{2}}(\mathrm{r}, \mathrm{t})\right) ; \quad \mathrm{t} \geq 0, \quad \mathrm{r} \in[0, \mathrm{R}]
$$

em que:

$$
\mathrm{r} \text { - coordenada esférica radial, } \mathrm{m}
$$$$
\mathrm{R} \text { - raio da fruta, } \mathrm{m}
$$

Neste modelo, a condição inicial é dada por:

$$
\mathrm{T}(\mathrm{r}, 0)=\mathrm{T}_{0} ; \quad \mathrm{r} \in[0, \mathrm{R}]
$$

e as condições de contorno são:

$$
\frac{\partial \mathrm{T}}{\partial \mathrm{r}}(0, \mathrm{t})=0, \quad \mathrm{t} \geq 0,
$$

e

$$
\mathrm{k}_{\mathrm{p}} \frac{\partial \mathrm{T}}{\partial \mathrm{r}}(\mathrm{R}, \mathrm{t})=\mathrm{h}_{\mathrm{c}}\left[\mathrm{T}_{\mathrm{s}}(\mathrm{t})-\mathrm{T}_{\mathrm{a}}(\mathrm{t})\right], \quad \mathrm{t} \geq 0
$$

Uma alternativa prática para a busca da solução da equação diferencial parcial (4) sujeita às condições (5), (6) e (7), é o uso de métodos numéricos baseados nos métodos de diferenças finitas, volumes finitos ou elementos finitos.

No caso do modelo unidimensional, entretanto, basta usarse de um esquema baseado no método de diferenças finitas, o que foi implementado segundo um esquema implícito.

O esquema implícito do método de diferenças finitas selecionado consiste em discretizar a equação (8) sujeita às condições (9), (10) e (11) de acordo com a convenção:

$$
\mathrm{T}_{\mathrm{i}}^{\mathrm{n}}=\mathrm{T}(\mathrm{i} \Delta \mathrm{r}, \mathrm{n} \Delta \mathrm{t}) ; \quad \text { para } \mathrm{i}=1, \ldots \mathrm{nx} \quad \mathrm{e} \quad \mathrm{n}=1, \ldots \mathrm{nt}
$$

em que:

i - define a localização dos pontos na direção radial

$\mathrm{nx}$ - define o último ponto na direção radial

$\Delta \mathrm{r}$ - é a distância entre os pontos da malha na direção radial

n - é o número total de passos de integração no tempo

$\Delta \mathrm{t}$ - é o tamanho do passo de integração no tempo, min

nt - define o último passo no tempo

Consideram-se as seguintes aproximações para os termos da equação (8), com ordens de aproximação o( $\Delta \mathrm{t})$; o $(\Delta \mathrm{r})$ e $\mathrm{o}\left(\Delta \mathrm{r}^{2}\right)$ respectivamente:

$$
\begin{aligned}
& \left.\frac{\partial T}{\partial t}(r, t)\right)_{i}^{n} \cong\left(\frac{T_{i}^{n+1}-T_{i}^{n}}{\Delta t}\right), \\
& \left.\frac{\partial T}{\partial r}(r, t)\right)_{i}^{n+1} \cong\left(\frac{T_{i+1}^{n+1}-T_{i}^{n+1}}{\Delta r}\right), \\
& \left.\frac{\partial^{2} T}{\partial r^{2}}(r, t)\right)_{i}^{n+1} \cong\left(\frac{T_{i-1}^{n+1}-T_{i}^{n+1}+T_{i+1}^{n+1}}{\Delta r^{2}}\right),
\end{aligned}
$$

além de formas análogas para os termos das condições iniciais e de contorno (9), (10) e (11). 
Essas aproximações transformam o problema de equação diferencial em um sistema tridiagonal de equações lineares que, a cada instante, deve ser resolvido para gerar a temperatura próxima ao centro das frutas; para tanto, uma estimativa do valor de $h_{c}$ deve ser inserida no modelo, sendo todos os outros valores de parâmetros tomados com os já referidos. Desta forma, para diversos valores em torno da estimativa de $\mathrm{h}_{\mathrm{c}}$, são obtidos os resultados da temperatura os quais são comparados com os dados experimentais. Desta forma, a partir da análise dos resíduos gerados, determina-se o melhor valor de $h_{c}$.

$\mathrm{O}$ algoritmo estabelecido a partir destas considerações foi implementado no ambiente computacional científico MATLAB 6.5.

No caso do modelo tridimensional, para se resolver a equação (4) segundo o método dos elementos finitos, o volume $\mathrm{V}$ da esfera que define a fruta foi dividido em elementos tetraédricos de primeira ordem (Buchanan, 1995); assim, a temperatura no elemento $\left(\mathrm{T}^{\mathrm{e}}\right)$ é calculada como:

$$
T^{e}(x, y, z)=a^{e}+b^{e} x+c^{e} y+d^{e} z
$$

em que $a^{e}, b^{e}, c^{e}$ e de são os coeficientes das funções de base utilizadas nos quatro nós de cada elemento, conforme (17), e a função de interpolação é definida em (18).

$$
\begin{aligned}
& \mathrm{T}_{\mathrm{i}}^{\mathrm{e}}(\mathrm{x}, \mathrm{y}, \mathrm{z} ; \mathrm{t})=\sum_{\mathrm{i}=1}^{4} \mathrm{~N}_{\mathrm{i}}^{\mathrm{e}}(\mathrm{x}, \mathrm{y}, \mathrm{z}) \mathrm{T}_{\mathrm{i}}^{\mathrm{e}}(\mathrm{t}) \\
& \mathrm{N}_{\mathrm{i}}^{\mathrm{e}}(\mathrm{x}, \mathrm{y}, \mathrm{z})=\frac{1}{6 \mathrm{~V}^{\mathrm{e}}}\left(\mathrm{a}_{\mathrm{i}}^{\mathrm{e}}+\mathrm{b}_{\mathrm{i}}^{\mathrm{e}}+\mathrm{c}_{\mathrm{i}}^{\mathrm{e}}+\mathrm{d}_{\mathrm{i}}^{\mathrm{e}}\right)
\end{aligned}
$$

Utilizando-se a expressão (17) na formulação fraca da equação (4) e se considerando as funções de interpolação idênticas em cada nó i e nó j, obter-se-á a sua forma semidiscretizada no volume dos tetraedros $\mathrm{V}_{\mathrm{e}}$ e na sua superfície $\Gamma_{\mathrm{e}}$, que pode ser expressa por:

$$
\mathrm{A}_{\mathrm{e}} \frac{\partial \mathrm{T}_{\mathrm{i}}}{\partial \mathrm{t}}+\mathrm{B}_{\mathrm{e}} \mathrm{T}_{\mathrm{i}}=\mathrm{f}
$$

em que:

$$
\begin{aligned}
& \mathrm{A}_{\mathrm{e}}=\int_{\mathrm{V}_{\mathrm{e}}} \mathrm{N}_{\mathrm{i}} \mathrm{N}_{\mathrm{j}} \mathrm{dV} \mathrm{V}_{\mathrm{e}} \\
& \mathrm{B}_{\mathrm{e}}=\int_{\mathrm{V}_{\mathrm{e}}} \alpha \nabla \mathrm{N}_{\mathrm{i}} \nabla \mathrm{N}_{\mathrm{j}} \mathrm{dV} \mathrm{V}_{\mathrm{e}}+\int_{\Gamma_{\mathrm{e}}} \mathrm{h}_{\mathrm{c}} \mathrm{N}_{\mathrm{i}} \mathrm{N}_{\mathrm{j}} \mathrm{d} \Gamma_{\mathrm{e}} \\
& \mathrm{f}_{\mathrm{e}}=\int_{\mathrm{V}_{\mathrm{e}}} \mathrm{h}_{\mathrm{c}} \mathrm{T}_{\mathrm{a}} \mathrm{N}_{\mathrm{j}} d \mathrm{~V}_{\mathrm{e}}
\end{aligned}
$$

A derivada que aparece na equação (19) é aproximada pela diferença finita avançada (Heinrich, 1999), com passo de integração $\Delta \mathrm{t}_{\mathrm{n}}$, resultando em:

$$
\left(\mathrm{A}+\Delta \mathrm{t}_{\mathrm{n}} \mathrm{B}\right) \mathrm{T}^{\mathrm{n}+1}=\mathrm{AT}^{\mathrm{n}}+\Delta \mathrm{t}_{\mathrm{n}} \mathrm{f}_{\mathrm{n}}
$$

Considerando-se n o instante de tempo do cálculo, o ve- tor $\mathrm{T}^{\mathrm{n}+1}$ de temperaturas nos nós do volume do figo é obtido resolvendo-se (23) desde que as temperaturas em $\mathrm{T}^{\mathrm{n}}$ sejam sempre conhecidas ao longo do tempo de resfriamento. Inicialmente, a temperatura ambiente $19^{\circ} \mathrm{C}$ é adotada nos nós do interior do figo e a temperatura $0{ }^{\circ} \mathrm{C}$ é imposta na superfície. Ao fim de cada intervalo de tempo se atribuem os valores em $\mathrm{T}^{\mathrm{n}+1}$ a $\mathrm{T}^{\mathrm{n}}$ até que o critério de parada de estabilidade térmica (24) seja alcançado, instante em que se considera o figo resfriado.

$$
\frac{\left|\mathrm{T}^{\mathrm{n}+1}-\mathrm{T}^{\mathrm{n}}\right|}{\left|\mathrm{T}^{\mathrm{n}}\right|}<10^{-4}
$$

Para isto se desenvolveu um algoritmo que, implementado computacionalmente em linguagem de programação $\mathrm{C}++$, resulta na curva de resfriamento da fruta. Para cada simulação a temperatura inicial na superfície da fruta e a temperatura ambiente na câmara de resfriamento foram consideradas iguais a $0^{\circ} \mathrm{C}$.

\section{RESULTADOS E DISCUSSÃO}

Os dados de temperatura próximo ao centro das frutas ao longo do tempo de resfriamento rápido de ar forçado estão na Figura 1, enquanto na Figura $1 \mathrm{~A}$ se acham os dados obtidos experimentalmente e a curva de ajuste exponencial, adaptados de Dussán-Sarria (2003) e, na Figura 1B, se encontram os resultados simulados para o problema unidimensional e para distintos valores de $h_{c}: 17,18,19,20,21,22$ e $23 \mathrm{~W} \mathrm{~m}^{-2}{ }^{\circ} \mathrm{C}^{-1}$, na qual o vetor indica o sentido do aumento do valor de $h_{c}$. Ressalta-se que para a geração desta figura se mantiveram fixos todos os outros parâmetros, inclusive aqueles associados à discretização no tempo e no espaço os quais, por serem usados no esquema implícito, não requerem cuidados com a consideração da malha temporal como função da malha espacial, que constou de 128 nós ao longo do raio da fruta.

Na Figura 1A se observa que no início o ajuste não representa adequadamente o processo de resfriamento rápido; além disso, o valor calculado de $\mathrm{h}_{\mathrm{c}}$ foi de $18,8 \mathrm{~W} \mathrm{~m}^{-2}{ }^{\circ} \mathrm{C}^{-1}$, o que gerou o valor do resíduo 0,3538; já os resíduos gerados nas distintas simulações mostradas na Figura 1B, se acham na Figura 2A; analisando-se o que mostra esta figura, constata-se que o melhor valor de $h_{\mathrm{c}}$, no sentido dos mínimos quadrados, está em torno de $22,0 \mathrm{~W} \mathrm{~m}^{-2}{ }^{\circ} \mathrm{C}^{-1}$ e, em assim sendo, buscando-se maior precisão se realizaram simulações variando-se o valor de $h_{c}$ a cada 0,01 a partir de 21,5 até 22,5 W m ${ }^{-2}{ }^{\circ} \mathrm{C}^{-1}$, cujos resíduos são mostrados na Figura $2 \mathrm{~B}$, na qual se tem que os resíduos são da mesma ordem apresentando coincidência apenas na primeira casa decimal e que o valor de $\mathrm{h}_{\mathrm{c}}=22,0 \mathrm{~W} \mathrm{~m}^{-2}{ }^{\circ} \mathrm{C}^{-1}$ é o que gera menor resíduo: 0,2076, o que pode ser mais bem observado na Figura 3A, em que são mostrados todos os resultados dessas simulações e na qual se verifica que as curvas estão sobrepostas.

A Figura 3B mostra a comparação dos dados experimentais com os resultados numéricos da temperatura aproximadamente no centro da fruta, obtidos para o modelo 
A.

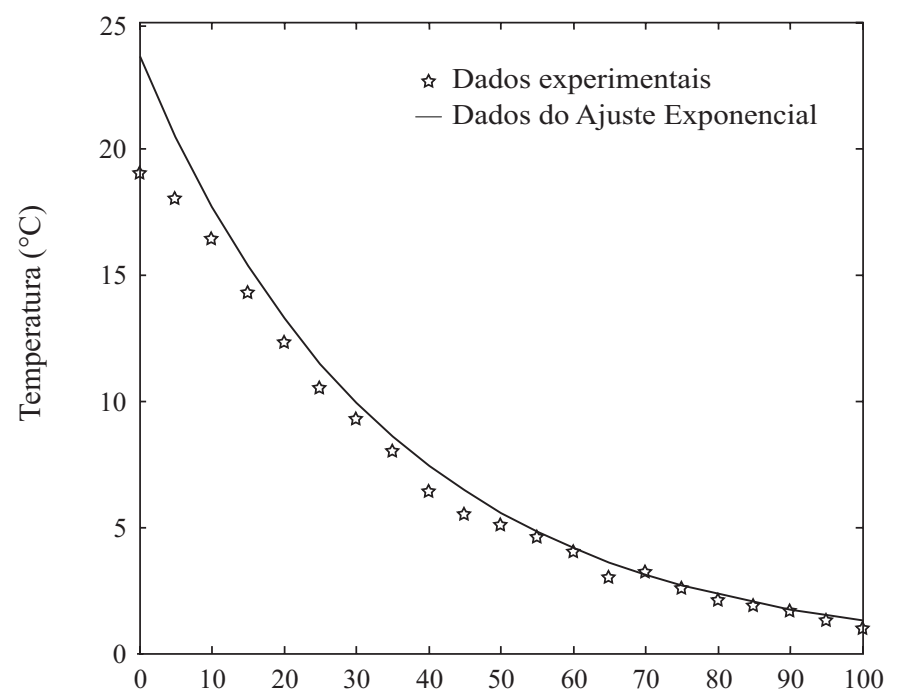

B.

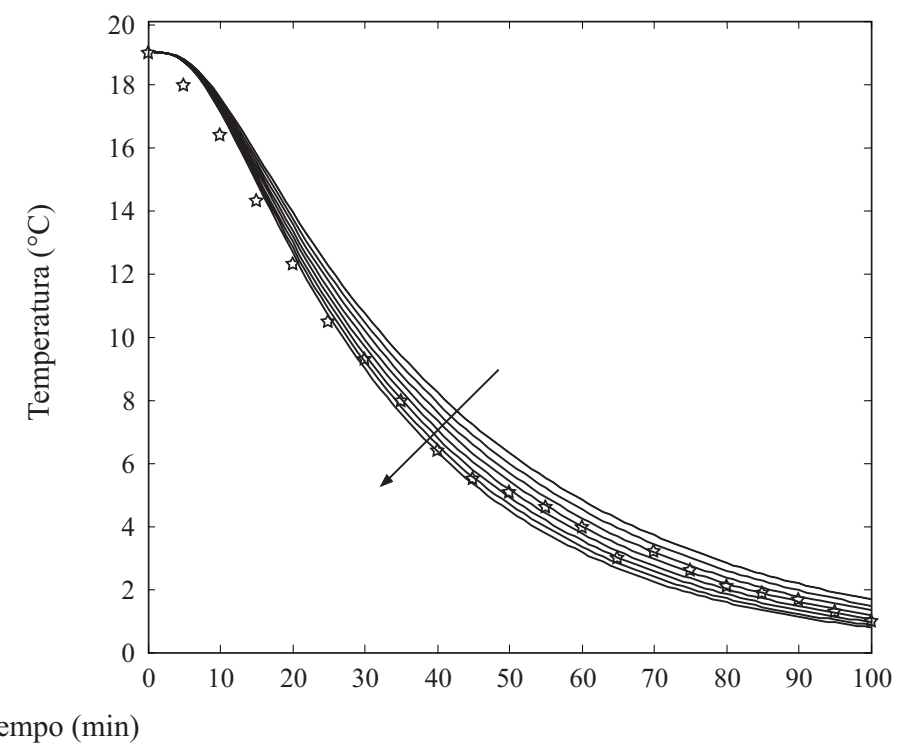

Figura 1. A. Dados experimentais $\left({ }^{*}\right)$ e curva de ajuste exponencial da temperatura $\left({ }^{\circ} \mathrm{C}\right)$ medida próximo ao centro dos frutos de figo durante o resfriamento rápido com ar forçado (linha contínua) B. Dados experimentais $\left({ }^{*}\right)$ e curvas de resfriamento rápido de figo obtidas da simulação numérica e se considerando distintos valores de para o problema unidimensional (linhas contínuas)

A.

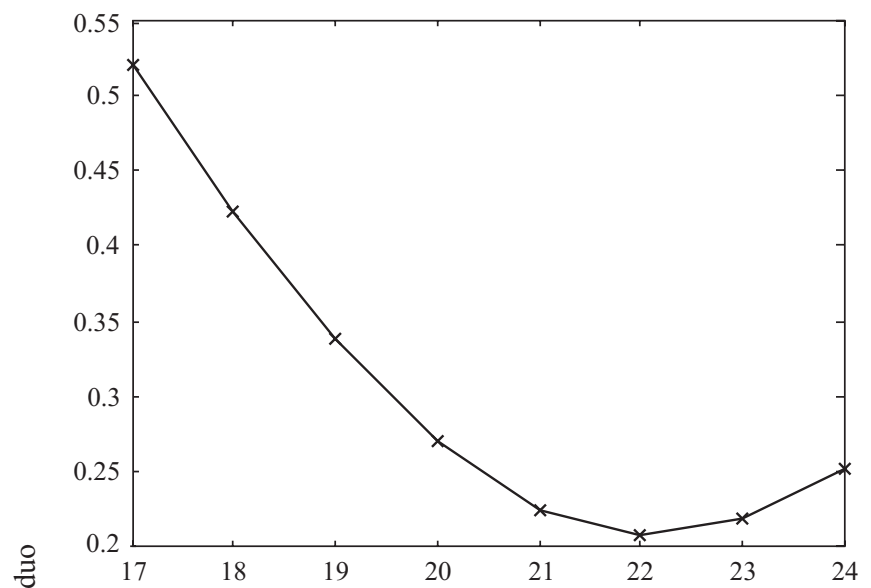

B.

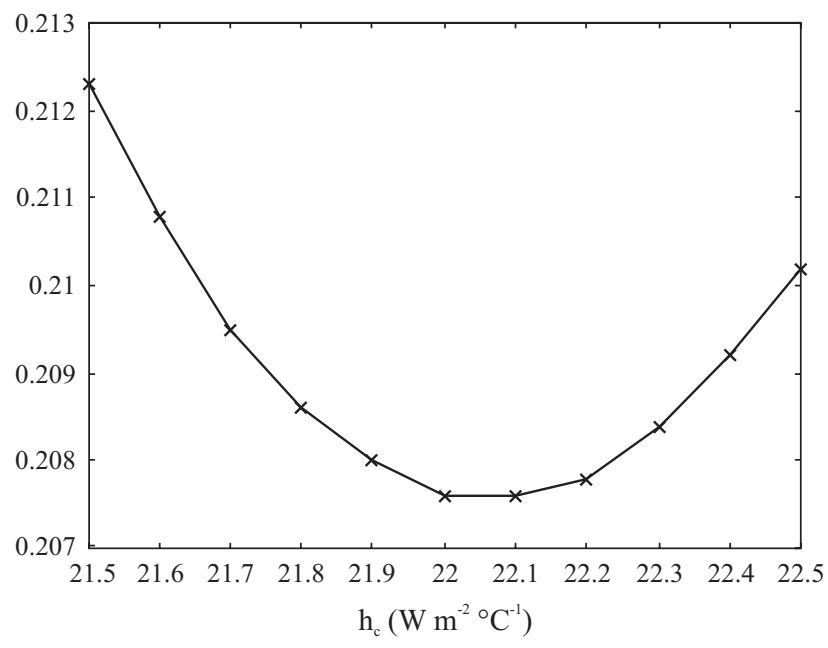

Figura 2. Resíduos obtidos das simulações numéricas mostradas na Figura 1B (A) e para valores variando a cada 0,01 e se iniciando em 21,5 até 22,5 $\mathrm{W} \mathrm{m}^{-2}{ }^{\circ} \mathrm{C}^{-1}(\mathrm{~B})$

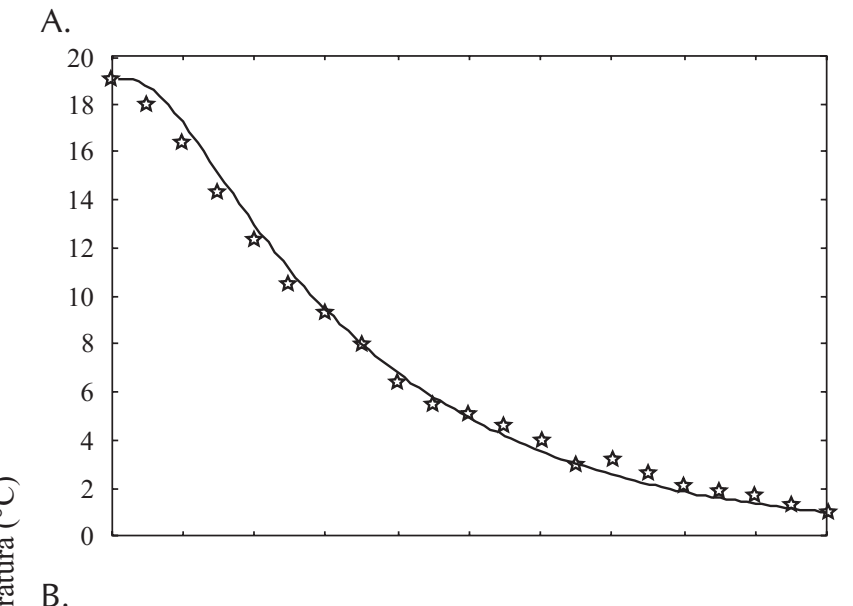

B.

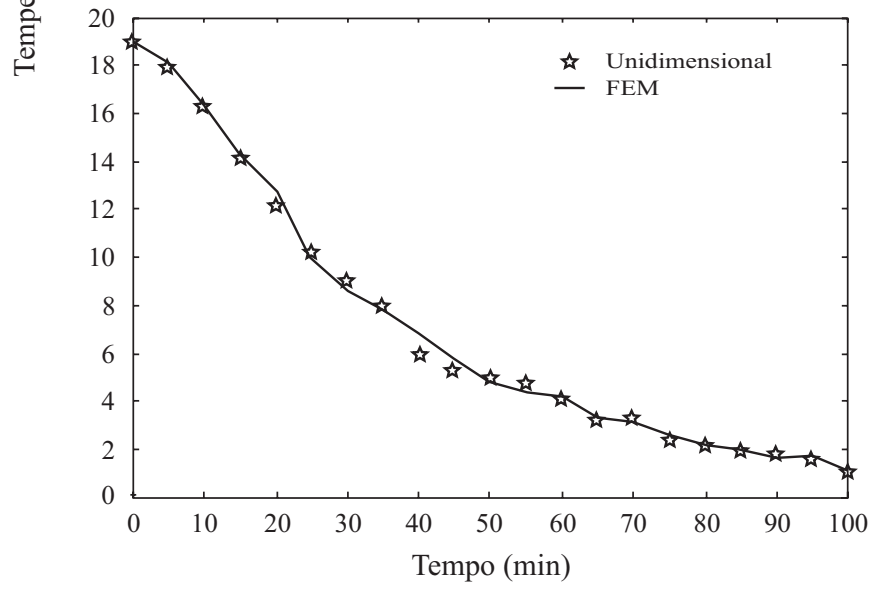

Figura 3. A. Comparação dos dados experimentais $\left({ }^{*}\right)$ com os resultados de todas as simulações numéricas para variando de 0,01 e se iniciando em 21,5 até $22,5 \mathrm{~W} \mathrm{~m}^{2}{ }^{\circ} \mathrm{C}^{-1}$ (linhas contínuas). B. Comparação dos dados experimentais $\left(^{*}\right.$ ) com os resultados obtidos ao longo do raio da fruta pelo modelo tridimensional, para o melhor valor de encontrado (linha contínua): $\mathrm{h}_{\mathrm{c}}=22,0 \mathrm{~W} \mathrm{~m}^{-1}{ }^{\circ} \mathrm{C}^{-1}$ 
tridimensional em vista do melhor valor de $\mathrm{h}_{\mathrm{c}}$ encontrado anteriormente: $\mathrm{h}_{\mathrm{c}}=22,0 \mathrm{~W} \mathrm{~m}^{-2}{ }^{\circ} \mathrm{C}^{-1}$. Ressalta-se, para o problema tridimensional, que o volume total da esfera de raio $\mathrm{R}=0,0285 \mathrm{~m}$ que define a região do domínio do problema foi dividido em 344 tetraedros, definindo, assim, a malha espacial de 63 nós.

\section{CONCLUSÕES}

1. Os resultados alcançados a partir do uso do modelo matemático e do método numérico, como especificados, são mais precisos que os obtidos do uso do ajuste exponencial e da expressão empírica na geração das curvas representativas do processo de resfriamento rápido com ar forçado.

2. Procurando o melhor valor de segundo os resultados oriundos do modelo matemático e do método numérico, conclui-se que o melhor valor de é $22,0 \mathrm{~W} \mathrm{~m}^{-2}{ }^{\circ} \mathrm{C}^{-1}$ (resíduo 0,2076).

3. Esta metodologia se mostra promissora para a continuidade de pesquisas de mesma natureza; entretanto, para determinação efetiva de seu valor e de sua interpretação, há necessidade de estudos complementares para avaliar a sensibilidade do valor de às condições experimentais às quais os figos foram submetidos.

4. A solução numérica do problema tridimensional gerou resultados equivalentes aos do modelo unidimensional, sugerindo que deve ser a utilizada para problemas semelhantes envolvendo geometrias de frutas mais complexas.

\section{AGRADECIMENTOS}

Os autores agradecem ao Professor Dr. Sylvio Luís Honório, da FEAGRI/UNICAMP, pela orientação na realização da Tese de Doutorado Dussán-Sarria (2003); à FAPESP (Fundação de Amparo à Pesquisa do Estado de São Paulo) pelo suporte financeiro no experimento, e aos comitês científicos dos CILAMCE/2005 e CNMAC/2006, pela oportunidade de discussão dos resultados.

\section{LITERATURA CITADA}

Amendola, M. Simulação numérica do processo de resfriamento rápido por ar forçado de limão. In: Congresso Nacional de Matemática Aplicada e Computacional 25, 2003. São José do Rio Preto: Anais... São José do Rio Preto: SBMAC, 2003a. p.143.

Amendola, M. Analysis of the parameters associated to the numerical simulation of the heat transfer process in agricultural products. In: International Workshop on Information Technologies and Computing Techniques for Agro-Food Sector-Afot. Barcelona, 2003, Barcelona. Abstracts... Barcelona: CIMNE, 2003b. p.151154.
Amendola, M. Sistemas de suporte a decisão para processos agrícolas: simulação numérica de processos de transferência de calor. In: Congresso Temático de Dinâmica e Controle da SBMAC, 3, 2004, Ilha Solteira. Anais... Ilha Solteira: UNESP, 2004. CD Rom

Amendola, M. Analysis of the parameters associated to the numerical simulation of the heat transfer in agricultural products. Tendências em Matemática Aplicada e Computacional, v.7, n.1, p.1-9, 2006a.

Amendola, M. Um algoritmo para a determinação do coeficiente de transferência de calor convectivo de frutos esféricos. In: Seminário Brasileiro de Produção Integrada de Frutas, 8, 2006, Vitória. Anais... Vitória: INCAPER, 2006b. p.237.

Amendola, M. Modelagem matemática e simulação numérica de processos de secagem e resfriamento de produtos agrícolas: metodologia, algoritmo e um exemplo prático. In: Seminário Internacional de Biomatemática, 3, 2006, Lima. Resumos... Lima: Universidad Nacional de San Marcos, 2006c. p.1.

Amendola, M.; Dussán-Sarria, S. Comparação de metodologias para a determinação do coeficiente convectivo de transferência de calor de frutas quando submetidas ao processo de resfriamento rápido com ar forçado: Estudo do caso de figos (Ficus carica L.). In: Iberian Latin-American Congress on Computational Methods in Engineering , 26, 2005, Guarapari. Proceedings... Guarapari: UFES, CILAMCE-2005, v.1, p.1-8.

Amendola, M.; Teruel, B. J. Esquema implícito e uso de splines para a simulação numérica do processo de resfriamento de frutas esféricas. Revista Brasileira de Engenharia Agrícola e Ambiental, v.9, n.1, p.78-82, 2005.

Buchanan, G. R. Schaum's outline of finite element analysis. New York: McGraw-Hill, 1995. 264p.

Dincer, I. Thermal cooling data for figs exposed to air cooling. International Communications Heat Mass Transfer, v.22, n.4, p.559-566, 1995.

Dussán-Sarria, S. Resfriamento rápido e armazenamento refrigerado do figo (Ficus carica L.) 'Roxo de Valinhos' e seus efeitos na qualidade da fruta. Campinas: FEAGRI/UNICAMP, 2003. 150p. Tese Doutorado

Dussán-Sarria, S.; Honório, S. L. Condutividade e difusividade térmica do figo (Ficus carica L.) 'Roxo de Valinhos'. Revista Brasileira de Engenharia Agrícola, v.24, n.1. p.185-194, 2004.

FAOSTAT. Organización de las Naciones Unidas para la Agricultura y la Alimentación. 2008. http://faostat.fao.org/. 9 Abr.2008.

Heinrich, J.C.; Pepper, D.W. Intermediate finite element method: fluid, flow and heat transfer. Philadelphia: Taylor \& Francis, 1999. 596p.

IBGE. Instituto Brasileiro de Geografia e Estatística. Ministério do Planejamento, Orçamento y Gestão. 2008. http:// www.ibge.gov.br/. 9 Abr.2008.

Mascheroni, R. H.; Calvelo, A. A. Simplified model for freezing time calculations in foods. Journal of Foods Science, v.47, p.1201-1207, 1982.

Novy, M.; Kieckbush, T. G. Equações para o cálculo do tempo de resfriamento em leitos de frutas esféricas. Ciência e Tecnologia de Alimentos, v.6, p.1-16, 1986.

Pirozzi, D. C. Z. Simulação Numérica do resfriamento rápido por ar forçado de morangos. Campinas: FEAGRI/UNICAMP, 2002. 82 p. Dissertação Mestrado 
Pirozzi, D. C. Z.; Amendola, M. Modelagem matemática e simulação numérica do resfriamento rápido de morango com ar forçado. Engenharia Agrícola, v.25, n.1, p.222230, 2005.

Rabello, A. A.; Amendola, M.; Dussán-Sarria, S. FEM Analysis of forced air precooling process of fig fruit (Ficus carica L.). In: Congresso Nacional de Matemática Aplicada e Computacional 29, 2006, Campinas. Anais... Campinas: SBMAC, 2006. CD Rom
Thompson, J. F.; Mitchell, F. G.; Rumsey, T. R.; Kasmire, R. F.; Crisosto, C. H. Commercial cooling of fruits, vegetables and flowers. Publication 21567, Oakland: University of California. 1998. 59p. Trelea, I. C.; Alvarez, G.; Trystram, G. Nonlinear predictive optimal control of a batch refrigeration process. Journal of Food Process Engineering, v.21, p.1-32, 1998.

Turk, R. Effects of harvest time and pre-cooling on fruit quality and cold storage Ficus carica L. Cv. 'Bursa syahjiu'. Acta Horticulturae, v.258, p.279-285, 1989.

R. Bras. Eng. Agríc. Ambiental, v.13, n.2, p.176-182, 2009. 